\title{
A Comparative Trial: The Safety and Clinical Efficacy of PEG 3350 and Liquid Paraffin in Management of Chronic Functional Constipation in Children
}

\author{
Abbaslou Parvin ${ }^{1 *}$, Abbaslou Farzaneh ${ }^{2}$, Hosseini Nasab $\mathrm{Ali}^{3}$ \\ ${ }^{1}$ Department of Pediatric Gastroenterology, Afzalipour Hospital, Kerman University of Medical Sciences, Kerman, Iran; ${ }^{2}$ Depatment \\ of Pediatrics, Afzalipour Hospital, Kerman University of Medical Sciences, Kerman, Iran; ${ }^{3}$ Department of Pediatric Infectious Dis- \\ ease, Afzalipour Clinical Research Unit, Kerman University of Medical Sciences, Kerman, Iran. \\ Email: "parvin_abbaslou@yahoo.co.uk
}

Received May $26^{\text {th }}, 2012$; revised June $28^{\text {th }}, 2012$; accepted July $14^{\text {th }}, 2012$

\begin{abstract}
Background: Constipation is one of the most common problems in the pediatric age group. In the vast majority, chronic constipation is idiopathic or functional. In addition to education and behavioral changes, disimpaction and maintenance treatment with medications are the main pillars of successful outcome. The medications are variably effective and have potential side effects. Objectives: The current clinical trial was designed to evaluate clinical efficacy of polyethylene glycol (PEG) 3350 and liquid paraffin in chronic functional constipation. Besides, subjects were followed carefully for the side effects. Material and Methods: This study was involved 110 children suffering from chronic functional constipation. They were placed into two different groups, randomly. Each group was treated with PEG 3350 or liquid paraffin. The clinical efficacy and side effects were monitored. Results: After disimpaction with bisacodyl suppositories or combined with Mineral oil enemas in hard fecal impaction, maintenance therapy with PEG 3350 or liquid paraffin was started. The treatment duration and efficacies were not different between the two groups. Furthermore, serious adverse drug reaction was not observed. The family history of constipation and presence of incontinence were two worse prognostic factors. Conclusion: No difference was observed between PEG 3350 and liquid paraffin in chronic functional constipation in children in terms of their efficacies. They are safe and effective medications in this age group.
\end{abstract}

Keywords: Children; Constipation; Liquid Paraffin; Polyethylene Glycol

\section{Introduction}

Constipation is a common disorder among children; as many as $25 \%$ of visits to pediatric gastroenterologists are for the treatment of this condition. Chronic constipation in children often doesn't have any organic cause and called functional. From 2006, ROME III Diagnostic Criteria have been defined to unify the functional constipation definitions [1-3].

Functional constipation may present with difficult and infrequent defecation or incontinence. Urinary problems, psychological disorders such as low self-esteem and depression, chronic abdominal pain and decreased appetite are other common presentations $[1,2]$.

NASPGHAN recommended four important steps for treatment of functional constipation: education, disimpaction, prevention of rectal reimpaction and regular follow up. Various disimpaction protocols using oral or

"Corresponding author. rectal routs have been published [4-6]. After disimpaction, variety of laxatives for restoring a regular defecation pattern and preventing relapses has been used $[1,2]$.

Polyethylene glycols (PEG) are osmotic and nonabsorbable compounds which are not metabolized by colonic bacteria. PEG 3350 without electrolytes is available as a powder and widely used to treat childhood constipation because of its effectiveness, safety and palatability [1].

Liquid paraffin is the most used lubricant laxative and composed of saturated hydrocarbons obtained from petroleum. Lipoid pneumonia is the most serious complication and a reason to avoid administration to patients with tendency for pulmonary aspiration $[1,2]$.

This study was aimed to compare the clinical efficacy and side effects of these two commonly used laxatives in childhood functional constipation.

\section{Material and Mothods}

The current trial was performed from April 2010 until 
March 2011 in Afzalipour Hospital on 156 constipated children who obtained ROME III Criteria. After education, a booklet including all necessary information as well as a symptom and medication diary for every month of treatment were provided to each parent. Disimpaction was managed with pediatric suppositories of bisacodyl (5 $\mathrm{mg}$ ) two times a day or the rectal bisacodyl in combination with liquid paraffin enemas at a dose of $10 \mathrm{~mL} / \mathrm{Kg}$ body weight/dose (maximum $150 \mathrm{~mL}$ ) two times a day in hard fecal impaction.

Following successful disimpaction, patients received PEG 3350 or liquid paraffin randomly as a maintenance treatment. The questionnaire regarding demographic data, growth measures, symptoms, past medical and family histories and physical examination findings were recorded. The children were followed through periodic appointments while the dose adjustment was advised to yield one or two soft stools daily and the side effects were monitored. When behavioral and dietary changes were established and normal bowel pattern $(\leq 2$ smears in a month, $\geq 3$ bowel movements in a week without abdominal pain during maintenance therapy) were achieved for a few months, the investigator decided to discontinue the medication gradually. In addition, one month after the complete discontinuation, patients were visited for relapses. In fact, they would be considered as a complete recovery case if they didn't experience any relapse without medication. If so, they called successfully treated subjects and needed to continue the medication longer. Moreover, during the follow up, retractable cases were worked up for organic causes and the case would be excluded from the study if an organic etiology was found.

The collected data were analyzed by SPSS V16 software. Furthermore, all criteria were carried out with $\alpha=$ 0.05 level of significance.

\section{Findings}

In this trial, 156 children aged 9 months to 11 years were enrolled. 24 cases were excluded due to absence of timely manner follow up and early cessation of medications. 17 retractable cases proceeded with further studies for organic causes and didn't complete the trial because of finding organic etiologies. These secondary etiologies included: hypocalcemia in 1, hypokalemia in 1, hypothyroidism in 2 , cow's milk protein allergy in 2 , ultra short Hirshprung disease in 2, and anterior ectopic anus in 8 subjects.

The rest of patients, 110 cases with mean age $36.76 \pm$ 26.94 months, completed the study. They were 59 girls and 51 boys. Constipation began in mean age 19.62 (2 78 ) months. Recorded symptoms included: withholding behavior in $93.6 \%$, decreased appetite in $62.5 \%$, chronic abdominal pain in $34.5 \%$ and incontinence in $25 \%$ of cases. Moreover, urinary problems were reported in $21.8 \%$; the most common was urinary tract infection. The family history of constipation was positive in $22.7 \%$ of subjects. Children nutritional status was assessed according to Gomez and Waterlow Criteria: $45.5 \%$ of all patients were mildly underweighted and $6.4 \%$ of them were mildly stunted. Anogenital index was $0.44 \pm 0.09$ $(0.35-0.65)$ in girls and $0.51 \pm 0.11(0.40-0.60)$ in boys. What's more, anal fissure in $62.7 \%$ and rectal fecal impaction in $35.5 \%$ of children were detected in the first examination.

All cases with fecal impaction were free of impaction in maximum of 5 days while $7.27 \pm 2.25$ doses of paraffin enemas and rectal bisacodyl were administered. Next, cases were randomly divided into two groups for maintenance treatment; the baseline characteristics of either group were balanced. Fifty one cases received PEG 3350 with initial mean dosage $0.6 \pm 0.16 \mathrm{~g} / \mathrm{Kg}$ body weight/day which was altered to optimal mean dosage $0.64 \pm 0.24$ $\mathrm{g} / \mathrm{Kg} /$ day. In the next group, 59 children used liquid paraffin. The starting paraffin dosage was $0.52 \pm 0.15$ $\mathrm{mL} / \mathrm{Kg} /$ day and further adjustment resulted in $0.86 \pm$ $1.53 \mathrm{~mL} / \mathrm{Kg} /$ day.

In PEG group, 37 cases $(73 \%)$ obtained complete recovery, and 14 patients $(27 \%)$ were treated successfully. In liquid paraffin group, 38 cases (64\%) recovered completely, 19 cases $(32 \%)$ were treated successfully and 2 children (4\%) didn't respond (Table 1). There was no difference between the two groups in terms of full recovery or successfully treatment rates $(\mathrm{P}$ Value $=0.333$ ).

The length of the drug therapy to obtain successful treatment in PEG group was $6.44 \pm 3.05$ months and in liquid paraffin group was $6.96 \pm 2.76$ months without any statistical difference $(\mathrm{P}$ Value $=0.355)$. The treatment duration to complete recovery was $5.89 \pm 2.60$ months in PEG group and $6.27 \pm 2.53$ months in paraffin group. The difference wasn't statistically meaningful (P Value $=0.119$ ).

Recovery rate in continent patients was higher than incontinent subjects ( $\mathrm{P}$ Value $<0.0001)$. Furthermore, recovery rate was lower in children with positive family history of chronic constipation in first-degree relatives (P Value $<0.0001)$. In addition, the recovery rate didn't

Table 1. The comparison of PEG and liquid paraffin efficacy in childhood functional constipation.

\begin{tabular}{lccc}
\hline \multirow{2}{*}{ Drug effect } & \multicolumn{2}{c}{ Drug } & \multirow{2}{*}{ P Value } \\
\cline { 2 - 3 } & Paraffin & PEG & \\
\hline Treatment Failure & $2(4 \%)$ & 0 & 0.333 \\
Successful Treatment & $19(32 \%)$ & $14(27 \%)$ & 0.333 \\
Complete Recovery & $38(64 \%)$ & $37(73 \%)$ & 0.333 \\
Total & $59(100 \%)$ & $51(100 \%)$ & \\
\hline
\end{tabular}


have any differences in children less or more than 2 years old and between each different sexes ( $\mathrm{P}$ Value $>0.05$ ).

Considering the side effects, diarrhea in $9.8 \%$, abdominal pain in $9.8 \%$, vomiting in $2 \%$ and dark color stool in $2 \%$ of patients in PEG group were recorded. Oil seepage in $25.4 \%$, diarrhea in $10.7 \%$ and abdominal pain in $5.4 \%$ of liquid paraffin-treated cases occurred.

In PEG group, only 30 cases signed the written consent forms for doing some biochemical blood tests including blood sugar, $\mathrm{Na}, \mathrm{K}, \mathrm{Ca}, \mathrm{P}, \mathrm{BUN}, \mathrm{Cr}$, SGOT and SGPT at the end of the medical treatment. All of the blood works were reported normal except mildly elevation of SGPT in 2 cases.

\section{Discussion}

Laxatives are the most important part in constipation management. Although some authors mentioned that there is no sufficient data for supporting better laxatives efficacy than placebo, the growing body of literature recommends these medications $[1,2,7]$.

Liquid paraffin with high compliance rate has a few limitations especially in a patient with pulmonary aspiration risk $[1,2,8]$. Recently, Polyethylene glycols have received a great deal of attention due to effectiveness, palatability and safety and they were also recommended for subjects who have failed or are intolerant to other medications [7,9]. Different doses, durations, and success rates were used depending on study protocols [9-15]. Although PEG has been suggested with a dose of 0.2 $1.5 \mathrm{gr} / \mathrm{Kg} /$ day, the starting dose of $1 \mathrm{gr} / \mathrm{Kg} /$ day with adjustment every 3 days was recommended by Rahhal et al. [1,5,11-15]. The success rate was reported 55 to more than $90 \%$ [10-14]. Long-term therapy with a mean duration of $8.4(3-30)$ months was reported to be effective in chronic childhood constipation [15]. In this study, 73\% of PEG group was completely recovered and $27 \%$ were successfully treated with a dose of $0.64 \mathrm{gr} / \mathrm{Kg} /$ day for about 6 months. Indeed, $27 \%$ of subjects needed longterm medication for normal bowel pattern but the recovery rate in almost two third of cases is completely convincing to suggest PEG considering the safety and few side effects observed in this trial. Moreover, the medication was tolerated well in subjects with the age of less than two years and this is in agreement with the results reported in few previous trials $[10,11]$. The biochemical blood tests were in the normal ranges even though few studies have stated some imbalances in these measurements and another didn't accept these values' changes $[16,17]$. In paraffin group, the success rates and treatment length were similar to the PEG group. In addition, optimal paraffin dosage was $0.86 \mathrm{~mL} / \mathrm{Kg} /$ day without any serious side effects and the most common side effect was oil seepage that could be annoying in some patients.
Current data bases were searched for comparative trials focusing on laxatives in children chronic constipation. In two studies, liquid paraffin efficacy was higher than Sena and lactulose $[8,18]$. Other studies were reported better PEG efficacy than lactulose and similar efficacy with Magnesium hydroxide [10,19]. Karami et al. performed a comparative trial on PEG and liquid paraffin in chronic childhood functional constipation and stated their efficacies were remarkable that it was compatible with this study, but they administered higher dosages of medications and didn't evaluate the short-term outcome after drug discontinuation [14].

Positive family history of chronic constipation was a worse prognostic factor in this study; it may be due to common genetic background and environmental factors in a family. Furthermore, the presence of incontinence was another poor prognostic factor in this study as it has been mentioned by some authors $[1,11]$.

\section{Conclusion}

Polyethylene glycol 3350 and liquid paraffin are safe and highly effective medications in pediatric functional constipation even during infancy. The positive family history of chronic constipation and incontinence were poor prognostic factors for complete recovery. One third of chronic constipated patients need to continue the medications for probably years. Future studies should focus on long-term outcome.

\section{REFERENCES}

[1] R. T. Rahhal and A. Uc, "Motility Disorders, Functional Constipation, in Walker's Pediatric Gastrointestinal Disorders," In: S. I. Kleinman, O. Goulet, et al., Eds., BC Decker Inc., Hamilton, 2008, pp. 675-682.

[2] V. Loening-Baucke, "Constipation and Encopresis, in Pediatric Gastrointestinal and Liver Disease," In: H. J. Wyllie, Ed., Saunders Elsevier, Philadelphia, 2006, pp. 177-191. doi:10.1016/B978-0-7216-3924-6.50015-9

[3] S. M. Mugie, M. A. Benninga and C. D. I. Lorenzo, "Epidemiology of Constipation in Children and Adults: A Ssystematic Review," Best Practice \& Research Clinical Gastroenterology, Vol. 25, No. 1, 2011, pp. 3-18. doi:10.1016/j.bpg.2010.12.010

[4] N. Youseff, J. M. Peters, W. Henderson, S. Shultz-Peters, D. K. Lockhart and C. Di Lorenzo, "Dose Response of PEG 3350 for the Treatment of Childhood Fecal Impaction," Journal of Pediatrics, Vol. 141, No. 3, 2002, pp. 410-414. doi:10.1067/mpd.2002.126603

[5] V. Tolia, "Use of a Balanced Lavage Solution in the Treatment of Fecal Impaction," Journal of Pediatric Gastroenterology and Nutrition, Vol. 7, No. 2, 1988, pp. 299301. doi:10.1097/00005176-198803000-00025

[6] V. Tolia, C. H. Lin and Y. Elitsur, "A Prospective Ran- 
domized Study with Mineral Oin and Oral Lavage Solution for Treatment of Faecal Impaction in Children," Alimentary Pharmacology \& Therapeutics, Vol. 7, No. 5, 1993, pp. 523-529.

doi:10.1111/j.1365-2036.1993.tb00128.x

[7] M. A. Pijers, M. M. Tabbers, M. A. Benninga and M. Y. Berger, "Currently Recommended Treatments of Childhood Constipation Are Not Evidence-Based, a Systematic Literature Review on the Effect of Laxative Treatment and Dietary Measures," Archives of Disease in Childhood, Vol. 94, No. 2, 2009, pp. 117-131. doi:10.1136/adc.2007.127233

[8] N. Urganci, B. Akyildiz and T. B. Polat, "A Comparative Study: The Efficacy of Liquid Paraffin and Lactulose in Management of Chronic Functional Constipation," Pediatrics International, Vol. 47, No. 1, 2005, pp. 15-19. doi:10.1111/j.1442-200x.2004.02001.x

[9] E. A. Bell and G. C. Wall, "Pediatric Constipation Therapy Using Guidelines and Polyethylene Glycol 3350," Annals of Pharmacotherapy, Vol. 38, No. 4, 2004, pp. 686-693. doi:10.1345/aph.1D297

[10] I. Voskuij, F. De lorijn, W. Verwijs, P. Hogeman, J. Heijmans, W. Mäkel, J. Taminiau and M. Benninga, "PEG 3350(Transipeg) versus Lactulose in the Treatment of Childhood Functional Constipation: A Double-Blind, Randomized, Controlled, Multicenter Trial," Gut, Vol. 53, No. 11, 2004, pp. 1590-1594. doi:10.1136/gut.2004.043620

[11] C. Dupont, B. Leluyer, F. Amar, N. Kalach, P. H. Benhamou, O. Mouterde and P. Y. Vannerom, "A Dose Determination Study of Polyethylene Glycol 4000 in Constipated Children: Factors Influencing the Maintenance Dose," Journal of Pediatric Gastroenterology and Nutrition, Vol. 42, No. 2, 2006, pp. 178-185. doi:10.1097/01.mpg.0000189349.17549.a9

[12] S. Nurko, N. N. Youssef, M. Sabri, A. Langseder, J. McGowan, M. Cleveland and C. Di Lorenzo, "PEG 3350 in the Treatment of Childhood Constipation: A Multicen- ter, Double-Blinded, Placebo-Controlled Trial," Journal of Pediatrics, Vol. 153, No. 2, 2008, pp. 254-261. doi:10.1016/i.jpeds.2008.01.039

[13] J. A. Dipalma, M. B. Cleveland, M. C. Gowan and J. L. Herrera, "A Comparison Polyethylene Glycol Laxative and Placebo for Relief of Constipation from Constipating Medications," Southern Medical Journal, Vol. 100, No. 11, 2007, pp. 1085-1090. doi:10.1097/SMJ.0b013e318157ec8f

[14] H. Karami, M. Khademlo and P. Niari, "Polyethylene Glycol Versus Paraffin for the Treatment of Childhood Functional Constipation," Iranian Journal of Pediatrics, Vol. 19, No. 3, 2009, pp. 255-261.

[15] D. S. Pashankar and W. P. Bishop, "Efficacy and Optimal Dose of Daily Polyethylene Glycol 3350 for Treatment of Constipation and Encopresis in Children," Journal of Pediatrics, Vol. 139, No. 3, 2001, pp. 428-432. doi: $10.1067 / \mathrm{mpd} .2001 .117002$

[16] D. I. Pina, M. M. Barba, O. S. Canton, M. A. Beltran, S. R. Ferreiro, R. V. Casas and J. L. Rey, "Safety and Efficacy of Polyethylene Glycol 3350 Plus Electrolyes for the Treatment of Functional Constipation in Children," Anales de Pediatría, Vol. 75, No. 2, 2011, pp. 89-95.

[17] S. H. Bae, "Long-Term Safety PEG 4000 in Children with Chronic Functional Constipation: A Biochemical Perspective," Korean Journal of Pediatrics, Vol. 53, No. 7, 2010, pp. 741-744. doi:10.3345/kjp.2010.53.7.741

[18] J. M. Sondheimer and E. P. Gervaise, "Lubricant versus Laxative in the Treatment of Chronic Functional Constipation of Children: A Comparative Study," Journal of Pediatric Gastroenterology and Nutrition, Vol. 1, No. 2, 1982, pp. 223-226. doi:10.1097/00005176-198201020-00012

[19] P. B. Gomes, M. A. Duarte and C. M. Mdo, "Comparison of the Effectiveness of Polyethylene Glycol 4000 without Electrolytes and Magnesium Hydroxide in the Treatment of Chronic Functional Constipation in Children," Journal of Pediatrics, Vol. 87, No. 1, 2011, pp. 24-28. 